\title{
Interpretation of sequence variants of the FBN1 gene: analog or digital? A commentary on decreased frequency of $F B N 1$ missense variants in Ghent criteria-positive Marfan syndrome and characterization of novel FBN1 variants
}

\author{
Yskert von Kodolitsch and Kerstin Kutsche \\ Journal of Human Genetics (2015) 60, 465-466; doi:10.1038/jhg.2015.95; published online 30 July 2015
}

$\mathrm{B}$ audhuin et al. ${ }^{1}$ report on their findings in B 292 individuals in whom sequencing of the FBN1 gene was performed. Their study reports 130 novel FBN1 variants, including phenotypic details and information on familial testing information, where they found a low frequency of $55 \%$ missense variants among the causative FBN1 sequence variants. The authors' major conclusion is that in addition to clinical criteria, genetic testing is important to establish Marfan syndrome. ${ }^{1}$ All this is important information, but the authors already provide an elaborate discussion of these issues. However, there is still another issue, which the authors did not comment on but which we think is important.

To the best of our knowledge, Baudhuin et al. are the first who apply the American College of Medical Genetics and Genomics (ACMG) standards and guidelines for the interpretation of sequence variants to $F B N 1$ sequence variants. ${ }^{2}$ The ACMG standards recommend a five-level classification system of sequence variants: pathogenic; likely pathogenic; variant of undetermined significance; likely benign; and benign. In their series, Baudhuin et al. identified a total of 280 FBN1 variants, of which they classified 207

$Y$ von Kodolitsch is at German Aorta Centre of Hamburg at the Centre of Cardiology and Cardiovascular Surgery of the University Medical Center Hamburg-Eppendorf, Hamburg, Germany; K Kutsche is at Institute of Human Genetics, University Medical Center HamburgEppendorf, Hamburg, Germany

E-mail: kodolitsch@uke.de as pathogenic or likely pathogenic (74\%), 33 as variant of undetermined significance $(12 \%)$ and 40 as likely benign or benign (14\%). In this brief comment, we will discuss why a five-level classification system of diagnostic findings is a serious problem for the clinical decision-makers, and we will refer to Anthony Wilden's classical essay on 'analog and digital communication' as theoretical framework for our argument. ${ }^{3}$

The authors of the ACMG guidelines state that their 'understanding of the clinical significance of any given sequence variant falls along a gradient, ranging from those in which the variant is almost certainly pathogenic for a disorder to those that are almost certainly benign'. ${ }^{2}$ This ACMG understanding of clinical significance corresponds to Wilden's analog form of information transmission. Analog information identifies a 'more or less' with continuous quantities that have no gaps between elements. ${ }^{3}$ In contrast to the analog form, the digital form of information transmission involves discrete elements and discontinuous scales with clear gaps between elements, positive and negative representations of quantities and dependency on 'zero'. Both forms, analog and digital deal with differences, where analog differences are differences of magnitude, frequency, distribution, pattern or organization, whereas digital differences are distinctions and oppositions, such as on/off firing of neurons, or yes/no in human communication. ${ }^{3}$ Therefore, only the digital form allows for precise distinctions, such as delineation of boundaries, inclusion and exclusion or agreement and disagreement.

Wilden argues that systems of communication usually employ both analog and digital communication. For example, a neuron receives and sums up analog quanta of information via the axon to either inhibit or release packets of acetylcholine which corresponds to either inhibition of firing or firing of the neuron. Therefore, neurons digitalize analog information and in doing so they reduce meaning but gain information. ${ }^{3}$ Wilden argues that translation of analog information to digital information typically happens not only at boundaries between systems, such as neuron and muscle, but also in communication between humans, ${ }^{3}$ or as we will argue, between different professional disciplines or different institutions.

The five-level classification system reflects the ACMG's understanding of clinical significance of sequence variants as gradient. Therefore, the ACMG suggests analog rather than digital interpretation of sequence variants. From an internal perspective of the molecular genetics/biological science, an analog interpretation appears compelling; molecular biology is complex, there are no gaps, but only differences of magnitudes. But how can an analog communication cross the system boundary of molecular biology to inform external systems such as physicians, clinical decision-makers or patients? 
Clinical decision-making is digital. Does the patient have Marfan syndrome? Will we recommend beta-blockers? Do we prohibit contact-sports? Do we perform elective replacement of the aortic root? There is a 'yes' or a 'no', with no gap between both. The clinician decides like a neuron: fire or not. In the digital world of decision-making, analog information must be ignored or digitalized. If the molecular scientist does not digitalize her/his interpretation of sequence variants, then somebody else has to try: the physician or the well-informed patient. The molecular expert, however, is the scientist rather than the clinician or the patient, and hence she/he has to do his or her best to digitalize the sequence interpretation. However, without any detailed clinical information, the molecular geneticist is not able to come to a 'yesno-decision'. Thus, the information from clinicians and patients need to be fully exploited to provide a digitalized sequence interpretation.

The same problem is well-known in the communication of diagnostic imaging findings; radiologists often find it difficult to provide digital interpretations when they struggle in the fog of analog findings on their images. Practice guidelines of the American College of Radiology account for this difficulty with the suggestion to provide both a descriptive, analog interpretation of images and a digital conclusion or diagnosis. ${ }^{4}$ Clearly, not every image or every sequence variant can be pressed into a yes/no scheme; but most can! By gathering all information available on a given sequence variant using freely available online tools, such as different pathogenicity prediction programs and databases on allele frequency for variants in the general population (for example, ExAc Browser and Exome Variant Server), an experienced molecular geneticist should be in a position to come to a clear interpretation in most of the cases. It is praiseworthy, that Baudhuin et al. followed the ACMG's recommendations, but it is even more praiseworthy that they actually digitalized their five-level classification system; they lumped the classes pathogenic and likely pathogenic in one, corresponding to 'yes', and the classes likely benign and benign into 'no'. Moreover, they reduced the number of 33 individuals with a variant of undetermined significance to 22 by fully exploiting the clinical and family information. They reduced biological meaning but they gained diagnostic information. Good teamwork between clinicians and molecular/human geneticists is necessary to digitalize molecular information. The reward is enhanced diagnostic information and improved clinical decision-making. And this is what diagnostic sequence interpretation is all about.

\section{CONFLICT OF INTEREST}

The authors declare no conflict of interest.

1 Baudhuin, L. M., Kotzer, K. E. \& Lagerstedt, S. A Decreased frequency of FBN1 missense variants in Ghent criteria-positive Marfan syndrome and characterization of novel FBN1 variants. J. Hum. Genet. 60, 241-252 (2015).

2 Richards, S., Aziz, N., Bale, S., Bick, D., Das, S., Gastier-Foster, J. et al. Standards and guidelines for the interpretation of sequence variants: a joint consensus recommendation of the American College of Medical Genetics and Genomics and the Association for Molecular Pathology. Genet. Med. 17, 405-424 (2015).

3 Wilden, A. System and Structure 155-93 (Tavistock Publications, London, UK, 1972).

4 National Guideline C. ACR Practice Guideline for Communication of Diagnostic Imaging Findings. (Agency for Healthcare Research and Quality (AHRQ) Rockville, MD). http://www.guideline.gov/content.aspx? $\mathrm{id}=32541$. Accessed 15 July 2015. 